\title{
Small-scale magnetic field dynamics on the Sun at high spatial and temporal resolution
}

\author{
N. Bello González ${ }^{1}$, O. Okunev ${ }^{1,2}$, and F. Kneer ${ }^{1}$ \\ 1 Institut für Astrophysik, Friedrich-Hund-Platz 1, 37077 Göttingen, Germany \\ e-mail: [nazaret;olok; kneer] @astro.physik.uni-goettingen.de \\ ${ }^{2}$ Central Astronomical Observatory of the Russian Academy of Sciences, Pulkovskoye chaussee 65/1, 196140 St. Petersburg, Russia
}

Received 25 June 2008 / Accepted 18 September 2008

\section{ABSTRACT}

\begin{abstract}
Aims. We present examples of the time evolution of phenomena occurring in the quiet Sun photosphere as seen from observations of high spatial and spectral resolution and with a time cadence of $23 \mathrm{~s}$.

Methods. A time sequence of about 5 min has been taken with the Göttingen Fabry-Perot spectropolarimeter at the VTT in the Fe I $6173 \AA$ A line. The data were reconstructed with speckle methods.

Results. With a polarimetric sensitivity of $2 \times 10^{-3} I_{\mathrm{c}}\left(\sigma_{\mathrm{B}}=2 \mathrm{G}\right)$, the formation of a bright point as seen from broadband and lineminimum intensities, as well as in magnetograms and Dopplergrams can be traced in detail. Other events, like a stable mixed-polarity configuration in the intergranules and the appearance of magnetic signature above granules in both dark and bright areas are presented.
\end{abstract}

Key words. Sun: magnetic fields - techniques: high angular resolution - techniques: polarimetric

\section{Introduction}

The magnetism and dynamics of the solar fine structure at the photospheric level still remain unclear. The polarisation signal strengthens and weakens in reaction to the granular convective processes. Magnetic fields undergo changes by advection, diffusion, and flux cancellation. Efforts are being made to perform spectropolarimetry of high sensitivity and high spatial, spectral and temporal resolution. We contribute to this field of research with some observational evidence of such processes occurring at small scales.

\section{Observations and data analysis}

The observations from the quiet Sun disc centre were taken on July 23, 2007, with the Göttingen FPI spectrometer/polarimeter (Bello González \& Kneer 2008, and references therein) at the VTT/Observatorio del Teide/Tenerife. They were supported by the Kiepenheuer Adaptive Optics System (KAOS, von der Lühe et al. 2003). Within a field of view (FOV) of $\sim 33^{\prime \prime} \times 25^{\prime \prime}$, the Fe I $6173 \AA$ line $\left(g_{\mathrm{L}}=2.5\right)$ was scanned with a step of $17.7 \mathrm{~m} \AA$. To further shorten the spectral scanning time, the polarimeter, originally based on two ferroelectric liquid crystals (FLCs, for details see Bello González \& Kneer 2008), was used in pure Stokes $V$ mode, i.e. a fixed $\lambda / 4$ retarder under $45^{\circ}$ with respect to the $0^{\circ}$ orientation of the $\lambda / 2$ FLC retarder and the $\lambda / 4$ FLC was removed from the beam. The efficiency in this case was $\varepsilon_{V}=0.91$, which after applying speckle reconstruction techniques (see Bello González \& Kneer 2008; Bello González et al. 2008, and references therein) and proper noise filtering, translates into a polarimetric sensitivity of $\sim 2 \times 10^{-3} I_{\mathrm{c}}\left(\sigma_{\mathrm{B}}=2 \mathrm{G}\right)$. A time sequence of $\sim 5 \mathrm{~min}$ with a cadence of $23 \mathrm{~s}$ was taken.
We calculate line-of-sight (LOS) components of the magnetic and the velocity field by applying the centre-of-gravity method (Rees \& Semel 1979) to the circularly polarised components. From response functions for velocity calculated for the $\lambda 6173 \AA$ line, we have obtained a proper linear combination of LOS velocity maps corresponding to two different heights in the atmosphere, i.e. to $100 \mathrm{~km}$ and $300 \mathrm{~km}$ (above $\tau_{5}=1$ ), that we denote by deep and high, respectively.

\section{Temporal evolution of small-scale structures}

The combination of high spatial, spectral and temporal resolution allows the detailed analysis of the small-scale magnetodynamic processes occurring in the quiet Sun photosphere. We have selected four examples from a large variety of phenomena and present their evolution in time as seen from broadband (speckle reconstructed) images of 0.'25 spatial resolution, magnetograms, line-minimum intensity and Dopplergrams (for deep and high layers) with 0.33 resolution. To remove the effects of the 5 min oscillations, smoothed velocity maps (with a 3". $8 \times 3$ ". 8 wide boxcar) were subtracted from the Dopplergrams. Furthermore, a subsonic filter with a $7.5 \mathrm{~km} \mathrm{~s}^{-1}$ cutoff was applied to the time sequences of images and maps.

\subsection{Formation of a bright point}

Figure 1 depicts the evolution in time (from left to right) of a forming bright point (BP) at the junction of intergranular lanes as seen (from top to bottom) in the broadband, LOS magnetic field $\left(B_{\mathrm{LOS}}\right)$, line-minimum intensities and LOS velocities $\left(v_{\mathrm{LOS}}\right)$ in high and in deep layers. In the Dopplergrams a positive velocity means a downward flow. Contours of $-50 \mathrm{G}$ (thick) and $+10 \mathrm{G}$ 


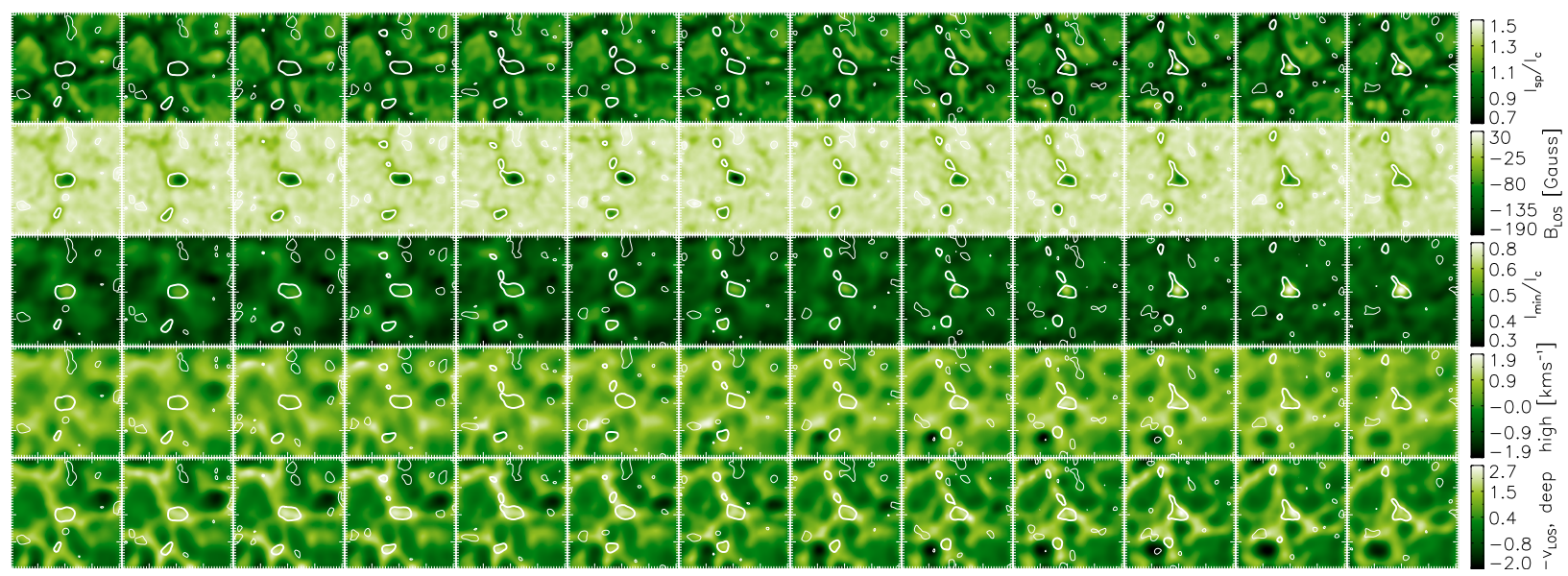

Fig. 1. Temporal evolution of bright point formation and surroundings. Time runs from left to right with $23 \mathrm{~s}$ cadence. From top to bottom: broadband, LOS magnetograms, line-minimum intensity, velocities in high and deep layers. Contours show $-50 \mathrm{G}$ (thick) and $+10 \mathrm{G}$ (thin) magnetic field strength levels. FOV is 4 "' $1 \times 4$.' 1 .

(thin) LOS magnetic field strengths are overplotted. The series consists of 13 consecutive scans with 23 s cadence. We note for the forming feature:

- Broadband: the formation of a BP at the junction of intergranular lanes already visible from early stages (centre of the FOV). The surrounding granules show a proper motion converging towards the forming structure. Contours show the distribution of the LOS magnetic signal along the intergranules and also above granules.

- $B_{\mathrm{LOS}}$ : the magnetic concentration associated with the BP shows an increasing field strength from $120 \mathrm{G}$ in the first scan up to $190 \mathrm{G}$ at the middle of the sequence. From then on, the LOS magnetic signal decreases while in both broadband and line-minimum intensities, the feature becomes brighter. The magnetic signal visible above the granule appears to be associated with the formation of a dark feature that appears in the first scan, later disappears and subsequently reappears. At the end of the sequence, when a dark granular dot is formed, there is no magnetic signal above $+10 \mathrm{G}(\widehat{=} 5 \sigma)$.

- Line minimum: a forming bright structure already can be seen at the beginning of the sequence in the centre of the FOV. Following similar behaviour to what is seen in broadband images, the maximum brightness is reached at the end of the sequence, when the magnetic field strength has decayed to $-60 \mathrm{G}$.

- $v_{\mathrm{LOS}}$, deep: the forming BP area shows an increasing downflow from $1.4 \mathrm{~km} \mathrm{~s}^{-1}$ (first scan) to $2 \mathrm{~km} \mathrm{~s}^{-1}$. In scan \#9, it suddenly decreases back to $1.4 \mathrm{~km} \mathrm{~s}^{-1}$, to later increase to $2.3 \mathrm{~km} \mathrm{~s}^{-1}$ in scan \#11. At the end of the sequence, it decreases again. The forming granular dark feature is at rest at the beginning of the sequence but later shows an up-flow to $0.5 \mathrm{~km} \mathrm{~s}^{-1}$ in the middle of the sequence and becomes a down-flow with a maximum of $0.8 \mathrm{~km} \mathrm{~s}^{-1}$.

- $v_{\text {LOS }}$, high: initially at rest, the area corresponding to the bright feature seen in broadband shows a down-flow gradually increasing to $1.2 \mathrm{~km} \mathrm{~s}^{-1}$ (scan \#9), to afterwards decrease again until becoming almost stationary. Meanwhile, the dark granular dot shows little dynamics around the zero LOS velocities in high layers. It starts as a down-flow, which turns into a small up-flow in the middle of the sequence and becomes as a down-flow when the dark feature is formed.

The origin of such bright structures is commonly associated with a convective collapse (Parker 1979) which causes the evacuation and therefore a brightening seen in the broadband images due to reduced opacity. We refer to Bello González et al. (2008) for a more detailed study of the evolution of these features.

\subsection{Evolution of mixed polarity magnetic concentration}

Figure 2 represents the evolution of a "stable" configuration of magnetic concentrations of opposite polarity. Following the structures marked by the contours of $|B|=40 \mathrm{G}$, one can see from top to bottom:

- Broadband: little evolution of granules with some small bright (with respect to the surroundings) features associated with the magnetic concentration. A brighter point with no strong magnetic signature can also be seen in the lower part; only after it disappeared is a signal of 20-25 G visible.

- $B_{\mathrm{LOS}}$ : the two magnetic concentrations of opposite polarity are separated by some 0.'5-0.'6 and show the same field strength, up to $75 \mathrm{G}$. The magnetic feature with opposite polarity decays almost completely within 120 s ( 5 scans), becoming stronger at the end of the sequence.

- Line minimum: bright structures appear during the decay of the magnetic features, better seen while the opposite-polarity magnetic feature decays.

- $v_{\text {LOS }}$, high: down-flows up to $0.8 \mathrm{~km} \mathrm{~s}^{-1}$ occur close to but not fully correlated with the magnetic structures. Such of magnetic structures appear to be better correlated with the borders of up and down-flows.

- $v_{\text {LOS }}$, deep: the strongest down-flows (up to $2.5 \mathrm{~km} \mathrm{~s}^{-1}$ ) appear correlated but somewhat displaced by $0.2-0.3$ from the magnetic features of opposite polarity.

This is an example of a mixed-polarity configuration in the range of $100 \mathrm{G}$ which remains stable in time. It lasts for more than $5 \mathrm{~min}$, throughout the sequence. Although strong dynamics occur in its close vicinity, no special proper motions are visible. One would expect the cancellation of magnetic flux, however some process behind this phenomenon maintains its stability.

\subsection{Temporal evolution of a splitting BP in an intergranular lane}

Figure 3 depicts the splitting process of a BP as seen in continuum. The contours here correspond to velocities of $+0.7 \mathrm{~km} \mathrm{~s}^{-1}$ 


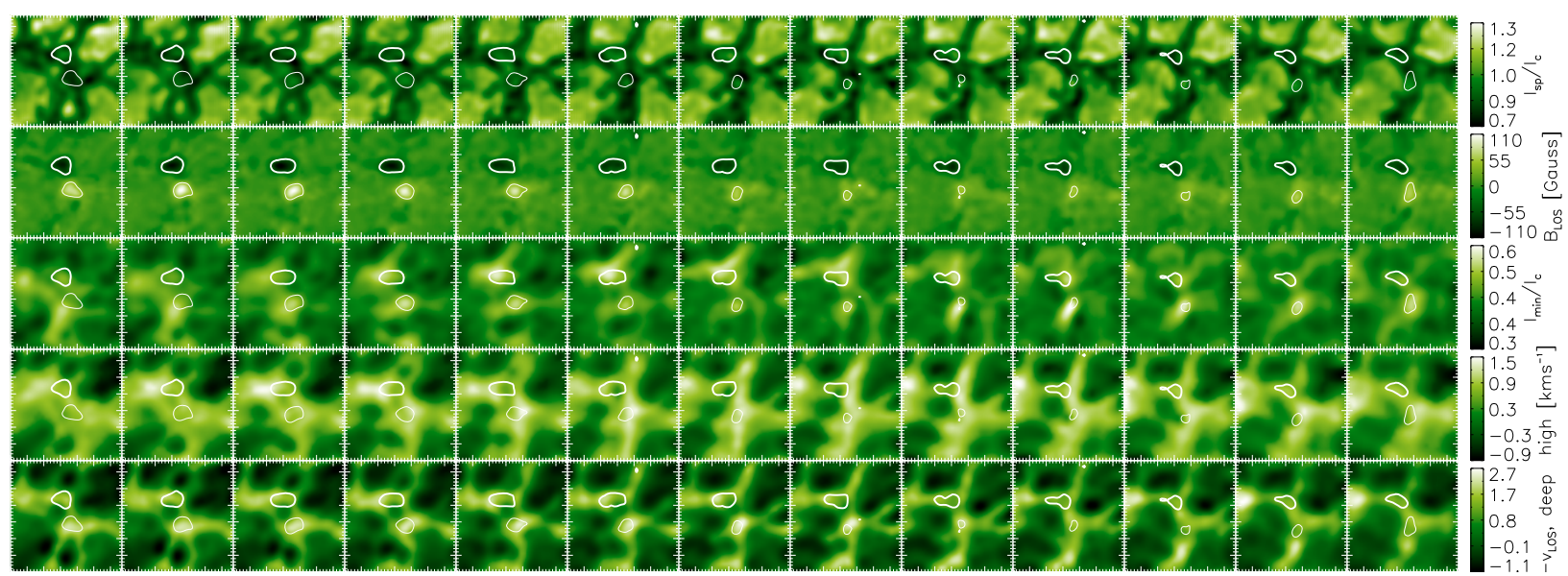

Fig. 2. Temporal evolution of a mixed-polarity magnetic concentration and surroundings. Same order as previous figure. Contours show $-40 \mathrm{G}$ (thick) and $+40 \mathrm{G}$ (thin) magnetic field strength levels. FOV is 3"' $4 \times 3$ "' 4 .

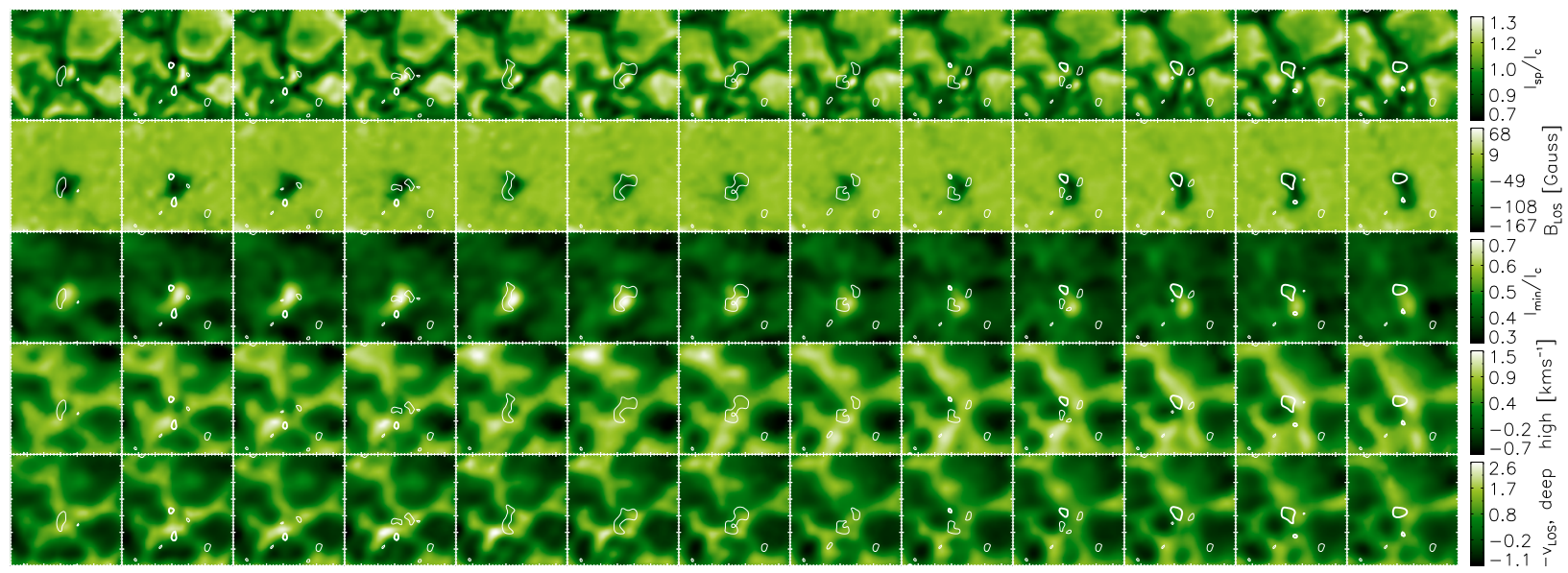

Fig. 3. Temporal evolution of a splitting BP in an intergranular lane. Contours represent velocities, $+0.7 \mathrm{~km} \mathrm{~s}^{-1}$ (thin) and $-0.6 \mathrm{~km} \mathrm{~s}^{-1}$ (thick), from the Stokes $V$ zero-crossing displacements. FOV is 3'. $4 \times 33^{\prime \prime} 4$.

(thin) and $-0.6 \mathrm{~km} \mathrm{~s}^{-1}$ (thick), obtained from displacements in the Stokes $V$ zero-crossing. From top to bottom:

- Broadband: temporal evolution of a bright feature in an intergranular lane splitting into two bright structures and separating during granular evolution. One becomes fainter and keeps its position and the brighter feature moves along the intergranular lane.

- $B_{\mathrm{LOS}}$ : strong magnetic concentration of negative polarity up to $170 \mathrm{G}$ decaying during the sequence and reinforced later. It appears strongly correlated to the bright intergranular features and becomes elongated following the morphology of those features. The remaining stronger fields during the decay appear concentrated between the BPs. At the end of the sequence the strongest fields remain better correlated with the structure that becomes fainter in continuum.

- Line minimum: a bright structure of some 0.'7 decaying in brightness with time is visible. It appears closely correlated with the magnetic structure as well as with the BPs seen in continuum, mainly with the brighter one.

- $v_{\text {Los }}$, high: the magnetic area appears correlated in the early stages with an area at rest. It turns into a down-flow during its evolution in the intergranule. The magnetic feature appears better correlated with the upward flow-downward flow (granule-intergranule) border.

- $v_{\text {Los }}$, deep: the magnetic area remains at rest.
The contours represent velocities from the Stokes $V$ zerocrossing, i.e. the magnetic areas. We notice unexpected up-flows (thick) surrounding the bright structures while down-flows (thin) occur in between the separating features. At late stages, the up-flow area becomes extended. It correlates with the fainter structure with a stronger magnetic signal and less brightness in higher layers. Compared with $v_{\text {LOS }}$ such up-flows appear better correlated with the upward flowing-downward flowing granular borders.

\subsection{Temporal evolution of magnetism in a granular area}

Figure 4 shows the temporal evolution of a granular area with a visible magnetic signal in both dark and bright granular regions. Also the evolution of a small BP along the intergranular lane can be followed. From top to bottom, one can see in the different maps with overplotted contours of LOS magnetic fields of $-20 \mathrm{G}$ (thick) and $+15 \mathrm{G}$ (thin):

- Broadband: an evolving granular region with a BP of $\sim 0$ '.32 moving along the intergranular lane following the perimeter of the upper granule.

- $B_{\mathrm{LOS}}$ : increasing magnetic signal (thick contour) associated with the BP with maximum values of up to $-160 \mathrm{G}$ in the middle of the sequence. It decays back to $-100 \mathrm{G}$ in the last 


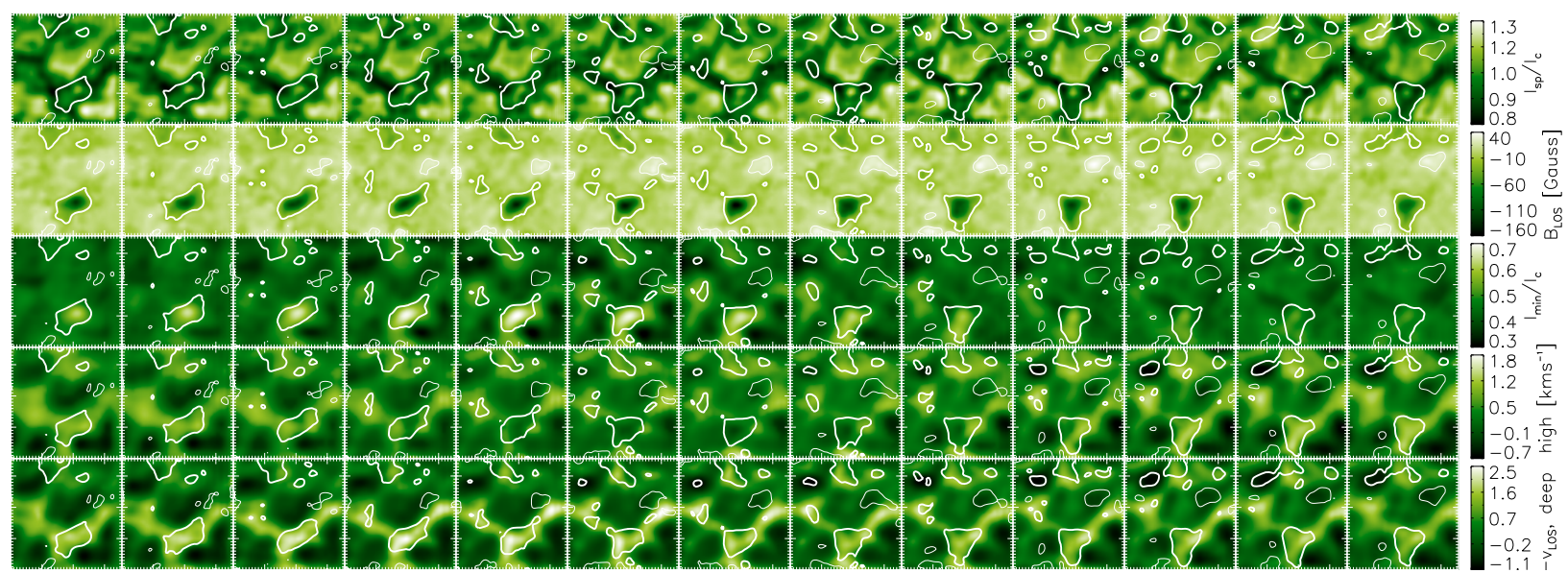

Fig. 4. Temporal evolution of a granular area with associated LOS magnetic signal. Contours show $-20 \mathrm{G}$ (thick) and $+15 \mathrm{G}$ (thin) magnetic field strength levels. FOV is 3.' $6 \times 3$ ".' 6 .

stage. An interesting feature is the appearance of a magnetic signal of opposite polarity, up to $+40 \mathrm{G}$, above the granules (thin contour).

- Line minimum: the most prominent feature is the brightness enhancement associated with the strongest magnetic field visible during the first half of the sequence. It starts to decrease in brightness when the LOS magnetic signal reaches its maximum, similarly to what was reported in Sect. 3.1.

- $v_{\text {LOS }}$, high: at the first stage of the sequence, the LOS velocities in high layers associated with the BP show a typical intergranular down-flow of up to $1 \mathrm{~km} \mathrm{~s}^{-1}$. It starts decreasing in time until becoming stationary in scan \# 7, when a downflow again appears gradually until $1 \mathrm{~km} \mathrm{~s}^{-1}$ at the end of the sequence.

- $v_{\text {LOS }}$, deep: the moving BP shows LOS velocities in deeper layers varying around $+1.3 \ldots+1.7 \mathrm{~km} \mathrm{~s}^{-1}$ (down-flows). The strongest down-flows (up to $+2.4 \mathrm{~km} \mathrm{~s}^{-1}$ ) occur in its vicinity and coincide in enhancement with the brightness seen in line-minimum intensities.

Strong displacements of the Stokes $V$ zero crossing occur near the BP from scan \# 5 on. At the end of the sequence, an up-flow of $-1 \mathrm{~km} \mathrm{~s}^{-1}$ appears to be closely correlated with the continuum BP. The signature of the LOS magnetic field of 10-20 G that we see in granules has been reported by Orozco Suárez et al. (2008) with data of the same quality but lower temporal resolution. It appears to be a common feature, better found during the formation of dark granular structures, but also found in bright granular areas.

\section{Conclusions}

We have reported on four events occurring in the quiet Sun photosphere. These are examples of the large variety of phenomena found in observations of high spatial and spectral resolution in a sequence taken with faster cadence than in previous studies. We have traced the formation of a BP in the junction of intergranular lanes which brightens in broadband after the associated magnetic signal ( $200 \mathrm{G})$ starts to decay. The forming feature shows temporally variable down-flows up to $2.3 \mathrm{~km} \mathrm{~s}^{-1}$.
We also described an intergranular mixed-polarity configuration lasting longer than $5 \mathrm{~min}$. Strong down-flows, up to $2.5 \mathrm{~km} \mathrm{~s}^{-1}$, appear close but not fully correlated to the feature of opposite polarity. The third example consists of a splitting bright feature in the intergranules whose major properties are downward and upward flows in its surroundings, only visible from velocities determined from the Stokes $V$ zero crossing. The last example shows the evolution of a magnetic feature moving along an intergranular lane. The formation of dark structures in granules, whose dynamics have been discussed by Hirzberger et al. (1999) also can be seen, here showing a weak magnetic signature prior to its formation. A magnetic signal of opposite polarity is also visible above the bright areas in granules as reported by Orozco Suárez et al. (2008).

With high angular resolution we could see, at small scales, interaction of magnetic fields with granular convection, influencing the structure and dynamics of the solar atmosphere as a whole. Our findings have implications for further studies of the small-scale magnetic fields in the Sun and of the physical processes underlying their influence on atmospheric dynamics. We have not yet reached the limits of angular resolution and polarimetric sensitivity to uncover the full richness of magnetoconvection in the stratified solar atmosphere.

Acknowledgements. N.B.G. and O.O. acknowledge financial support by Deutsche Forchungsgemeinschaft through grants KN 152/31-1 and KN 152/293 , respectively. The Vacuum Tower Telescope is operated by the KiepenheuerInstitut für Sonnenphysik, Freiburg, at the Spanish Observatorio del Teide of the Instituto de Astrofísica de Canarias.

\section{References}

Bello González, N., \& Kneer, F. 2008, A\&A, 480, 265

Bello González, N., Yelles Chaouche, L., Okunev, O., \& Kneer, F. 2008, A\&A, submitted

Hirzberger, J., Bonet, J. A., Vázquez, M., \& Hanslmeier, A. 1999, ApJ, 527, 405 Orozco Suárez, D., Bellot Rubio, L. R., del Toro Iniesta, J. C., \& Tsuneta, T. 2008, A\&A, 481, L33

Parker, E. N. 1979, Cosmical Magnetic Fields (Clarendon, Oxford)

Rees, D. E., \& Semel, M. D. 1979, A\&A, 74, 1

von der Lühe, O., Soltau, D., Berkefeld, T., \& Schelenz, T. 2003, SPIE, 4853, 187 